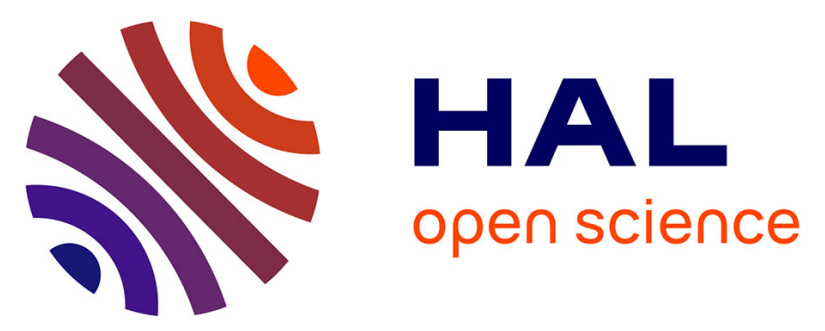

\title{
Gradient, non-gradient and hybrid algorithms for optimizing 3D forging sequences with uncertainties \\ Lionel Fourment
}

\section{To cite this version:}

Lionel Fourment. Gradient, non-gradient and hybrid algorithms for optimizing 3D forging sequences with uncertainties. Materials Processing and Design, Modeling, Simulation and Applications, NUMIFORM '07: 9th International Conference on Numerical Methods in Industrial Forming Processes, Jun 2007, Porto, Portugal. pp.Pages 475-480, 10.1063/1.2740856 . hal-00510567

HAL Id: hal-00510567

https://hal-mines-paristech.archives-ouvertes.fr/hal-00510567

Submitted on 1 Apr 2011

HAL is a multi-disciplinary open access archive for the deposit and dissemination of scientific research documents, whether they are published or not. The documents may come from teaching and research institutions in France or abroad, or from public or private research centers.
L'archive ouverte pluridisciplinaire HAL, est destinée au dépôt et à la diffusion de documents scientifiques de niveau recherche, publiés ou non, émanant des établissements d'enseignement et de recherche français ou étrangers, des laboratoires publics ou privés. 


\title{
Gradient, Non-Gradient and Hybrid Algorithms for Optimizing 3D Forging Sequences with Uncertainties
}

\author{
Lionel Fourment
}

CEMEF, Ecole des Mines de Paris, BP 207, 06904 Sophia Antipolis Cedex, France

\begin{abstract}
In the frame of computationally expensive 3D metal forming simulations, optimization algorithms are studied in order to find satisfactory solutions within less than 50 simulations and to handle complex optimizations problems with several extrema. Two types of algorithms are selected, which both utilize a meta-model to approximate the objective function and so reduce computational cost. This model either supports standard Evolutionary Algorithms, such as Genetic Algorithms, or is sequentially improved until finding a satisfactory and well approximated solution. The Meshless Finite Difference Method is the utilized meta-model, without (standard algorithm) or with (hybrid algorithm) the gradient information. This meta-model approach allows taking into account uncertainties on optimization parameters in an inexpensive way. The optimization procedure is modified accordingly. The proposed algorithms are first evaluated and compared on standard analytic functions, and then applied to a 3D forging benchmark, the shape optimization of preform tool in order to minimize the potential of fold formation.
\end{abstract}

Keywords: Optimization Algorithm, Response Surface, Uncertainties, Design of Experiments, Evolutionary Algorithm, Sequential Approximation, Shape Optimization, Tool Design, Forging

\section{INTRODUCTION}

One of the key issues of optimization in the frame of metal forming is the computational time required for a single simulation. More particularly, in the case of $3 \mathrm{D}$ forging, the non-steady nature of the large deformation process requires numerous remeshings and results into computational times ranging from several hours on a single computer machine to several days on a parallel computer. Therefore, it is not conceivable to carry out optimization procedures that would require too many simulations, which we have limited here to 50 . Under these circumstances, several authors have used gradient algorithms, and consequently turned toward the calculation of objective function gradients, either by the direct differentiation method [1-4] or by the adjoint state one $[5,6]$. On top of the complexity of these calculations, which require differentiating the finite element code equations, gradient algorithms only converge toward local minima, which can be sometimes satisfactory, but not always, as for instance in [7].

A first stage of robustness of an optimization algorithm is its ability to find a "global" minimum, by making it possible to screen the full parameters space and to check whether a satisfactory solution is achievable. The computational cost of raw "global" algorithms, such as Genetic Algorithms (GA), is generally a curb on their use. It proves necessary to use surrogate models to calculate the objective function for a very large number of parameter values. These models are referred to as Response Surfaces or Meta-models according to the frame in which they have been developed. They are based on different interpolation methods, such as Polynomial (mainly used in the Response Surface approach), Kriging [810] (mainly developed in a probabilistic context and then extended to deterministic data), Moving Least Square [11] (or Diffuse Element Method, which are derived from meshless interpolations), or else Meshless Finite Difference Method (MFDM) [12, 13] which is used here. They are utilized according two main manners [9]. Firstly, the meta-model can be substituted to the exact evaluations of the objective function within costly global algorithms, such as Evolution Strategies [8] or Genetic Algorithms [12]. Secondly, the meta-model can be utilized to build successive sequential approximations of the objective function to better and better approximate the global minimum. In this case, the algorithm usually starts from an initial Design of Experiments (DOE) that allows building a first approximation of the meta-model. Then, 


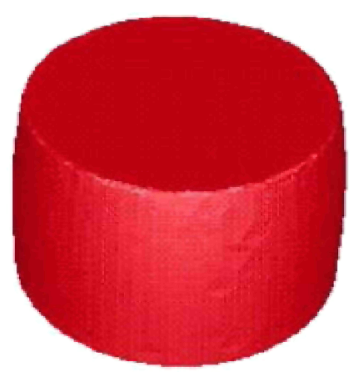

(a)

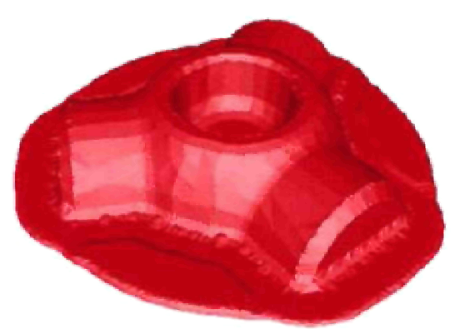

(b)

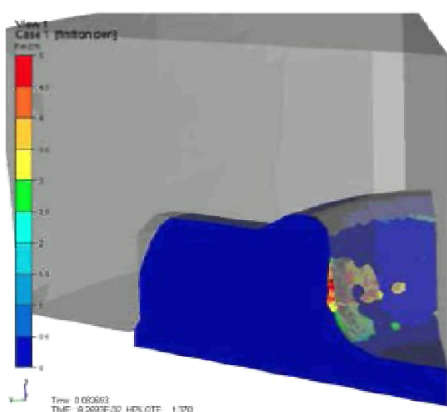

(c)

FIGURE 1. Initial billet (a) and forged spindle (b) at the end of forging. Lap in the colored zone (c) with initial preform.
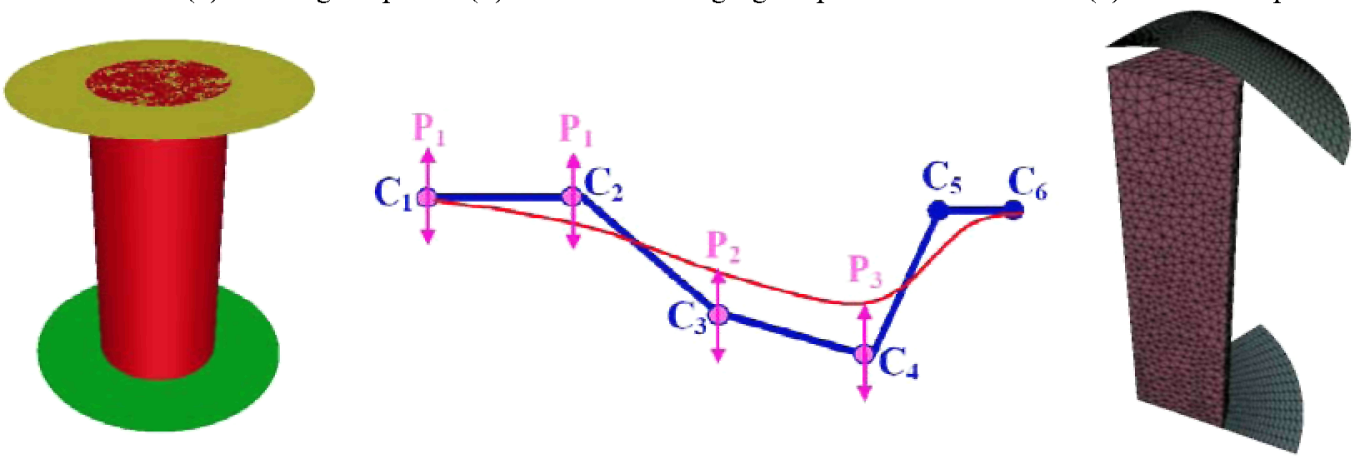

FIGURE 2. Initial design of preforming tool, parameterization of axisymmetric tool with Bspline functions, resulting tool.

additional points are introduced to improve the accuracy of the approximation, according to various strategies [9-11]

On the other hand, the second stage of robustness of an optimization algorithm results from taking into account the problem uncertainties, which may have a dramatic effect on the stability of the found solution.

This paper presents the two described strategies, which have been developed in the convenient case where the meta-model is derived from the MFDM. It allows using both non gradient and gradient interpolations, in which case the method may be called "hybrid" as it combines the gradient effect with an evolutionary algorithm. Gradients are calculated in the finite element software FORGE3® by the adjoint state method as presented in [6]. This approach allows taking into account uncertainties on optimization parameters, so providing a more robust algorithm. These algorithms are finally applied to a benchmark forging problem, the two-stepped forging of a $3 \mathrm{D}$ spindle.

\section{BENCHMARK OPTIMIZATION PROBLEM}

The benchmark problem is a simple but representative two-stepped sequence for forging a spindle from a cylinder (see Figure 1). The preforming operation is axisymmetric. The second and finishing one is closed-die forging with flash. With the initial design of the preforming dies, a flat design (see Figure 2-a), the material folds over during the second operation, as shown in Figure 1-c. This surface defect can be quantified by an unusual increase of the equivalent strain rate $\dot{\bar{\varepsilon}}$ on the free surface with respect to a reference value $\dot{\bar{\varepsilon}}_{\text {ref }}$. So, the following objective function provides a measure of the fold appearance potential:

$$
f(p)=\int_{t=0}^{t=t_{\text {end }}} \frac{1}{\left|\partial \Omega_{\text {free }}^{t}\right|}\left(\int_{\partial S_{\text {free }}^{t}}\left(\frac{\dot{\bar{\varepsilon}}}{\dot{\bar{\varepsilon}}_{\text {ref }}}\right)^{\alpha} d s\right)^{\frac{1}{\alpha}} d t(1)
$$

where $p$ represent the optimization parameters, $\partial \Omega_{\text {free }}^{t}$ the free surface at time $t$, and $\alpha$ a function parameter that is taken equal to 10 in the present applications. In the initial flat dies configuration, $f(p)=10.49$. The axisymmetric shape of the preforming tool is parameterized with a Bspline curve (see Figure 2) with 2 active parameters in the considered applications. The inevitable uncertainties on the parameter values should also be taken into account for such a problem. In fact, the objective 
function can be very sensitive to small variations of the preform shapes, and the tool shapes cannot be exactly machined according to design recommendations.

The computational cost for a single evaluation of the objective function is about 3 hours on a personal computer. The gradient of the objective function is calculated by differentiating the finite element software using the adjoint state method, as presented in [6].

\section{META-MODEL}

A meta-model allows calculating the surrogate function values (and possibly its gradients) from the exact values known at certain points referred to as "master" points. The present approach utilizes the Meshless Finite Difference Method (MFDM) [13].

\section{Meta-Model Using The Gradient Information}

For any point $i$ of the parameter space, located at $x_{i}$, the approximation $\tilde{f}_{i}$ of $f\left(x_{i}\right)$ is a function of $f_{v}=f\left(x_{v}\right)$ and $\nabla f_{v}=f\left(x_{v}\right)$ values of all $n_{v}$ master points $v$, which is obtained by the minimization (3) of the residues (4) of the set of first order Taylor series expansions written at any $x_{v}$ :

$$
\begin{gathered}
\forall v=1, n_{v}, \quad f_{i}=f_{v}+\nabla f_{v}\left(x_{i}-x_{v}\right)+O\left(\left\|x_{i}-x_{v}\right\|^{2}\right)(2) \\
\tilde{f}_{i}=\operatorname{MIN}_{\tilde{f}_{i}^{\prime}} \pi_{1}\left(\tilde{f}_{i}^{\prime}\right) \\
\text { where: } \pi_{1}\left(\tilde{f}_{i}\right)=\sum_{v=1, n_{v}} \frac{\left(\tilde{f}_{i}-f_{v}-\nabla f_{v}\left(x_{i}-x_{v}\right)\right)^{2}}{\left\|x_{i}-x_{v}\right\|^{4}} \\
\text { so: } \tilde{f}_{i}=\frac{1}{\sum_{v=1, n_{v}} \frac{1}{\left\|x_{i}-x_{v}\right\|^{4}} \sum_{v=1, n_{v}} \frac{\tilde{f}_{i}-f_{v}-\nabla f_{v}\left(x_{i}-x_{v}\right)}{\left\|x_{i}-x_{v}\right\|^{4}}} \text { (5) }
\end{gathered}
$$

\section{Meta-Model Without Gradient Information}

When the gradient of $\left(f_{v}\right)_{v=1, n}$ is not known, the Taylor series expansion is written in the inverse way, at $x_{i}$ for any $x_{v}$ master point:

$$
\begin{array}{cc}
\forall v=1, n_{v}, \quad f_{v}=\tilde{f}_{i}+\nabla \tilde{f}_{i}\left(x_{v}-x_{i}\right)+O\left(\left\|x_{v}-x_{i}\right\|^{2}\right) \\
\left(\tilde{f}_{i}, \nabla \tilde{f}_{i}\right)=\underset{\left(\tilde{i}_{i}^{\prime}, \tilde{f}_{i}^{\prime}\right)}{M I N} \pi_{0}\left(\tilde{f}_{i}^{\prime}, \nabla \tilde{f}_{i}^{\prime}\right)
\end{array}
$$

where: $\pi_{0}\left(\tilde{f}_{i}, \nabla \tilde{f}_{i}\right)=\sum_{v=1 n_{v}} \frac{\left(\tilde{f}_{i}+\nabla \tilde{f}_{i}\left(x_{v}-x_{i}\right)-f_{v}\right)^{2}}{\left\|x_{v}-x_{i}\right\|^{4}}(8)$

$\left(\tilde{f}_{i}, \nabla \tilde{f}_{i}\right)$ is then solution of the linear system:

$$
A\left(\begin{array}{c}
\tilde{f}_{i} \\
\nabla \tilde{f}_{i}
\end{array}\right)=b\left(\left(f_{v}\right)_{v=1, n_{v}}\right)
$$

$A$ can be inverted if there are sufficient and well localized master points $v$. In practice, there should be at least $(d+1)$ master points, where $d$ is the dimension of the parameter space. Practically, it is interesting to notice that the resolution of (9) also provides a surrogate gradient value $\nabla \tilde{f}_{i}$ at $x_{i}$.

\section{Approximation Error}

In both cases, the approximation error of $f_{i}$ by $\tilde{f}_{i}$ can be estimated by the following $\Delta \tilde{f}_{i}$ value:

$$
\Delta \tilde{f}_{i}=\sqrt{\left(\sum_{v=1, n_{v}}\left\|x_{i}-x_{v}\right\|^{-4}\right)^{-1} \pi\left(\tilde{f}_{i}\right)}
$$

$\Delta \tilde{f}_{i}$ makes it possible to compute an approximation of a lower bound of $f\left(x_{i}\right): \tilde{f}_{i}-\Delta \tilde{f}_{i}$. This value can be regarded as the best possible value of $f\left(x_{i}\right)$ suggested by the meta-model.

At the level of the entire parameter space $\Omega$, the approximation error $E$ is written as:

$$
E=\int_{\Omega}(\tilde{f}(x)-f(x))^{2} d x
$$

By substituting $\tilde{f}(x)$ by the value given by (5), or resulting from (9), a first order approximation of $E$ if written [14]:

$$
E\left(\left(x_{v}\right)_{v=1, n_{v}}\right)=\int_{\Omega} \frac{\sum_{v=1, n_{v}}\left\|x-x_{v}\right\|^{-2}}{\sum_{v=1, n_{v}}\left\|x-x_{v}\right\|^{-4}} d x
$$

$E\left(\left(x_{v}\right)_{v=1, n_{v}}\right)$ is an a priori error estimation of any function interpolated by the meta-model defined by the master points $\left(x_{v}\right)_{v=1, n_{v}}$.

\section{Design Of Experiments (DOE)}

The minimization of $E\left(\left(x_{v}\right)_{v=1, n_{v}}\right)$ with respect to the $n_{v}$ master points $\left(x_{v}\right)_{v=1, n_{v}}$ provides the best 
initial Design Of Experiments (DOE) for the considered meta-model, in a fully consistent manner:

$$
E\left(\left(x_{v}\right)_{v=1, n_{v}}\right)=\underset{x_{v}{ }^{\prime} \in \Omega^{n_{v}}}{\operatorname{mN}} E\left(\left(x_{v}{ }^{\prime}\right)_{v=1, n_{v}}\right)
$$

If there already exist $n_{u}$ master points $\left(x_{u}\right)_{u=1, n_{u}}$, the a priori interpolation error $E\left(\left(x_{u}\right)_{u=1, n_{u}},\left(x_{v}\right)_{v=1, n_{v}}\right)$ resulting from the addition of $n_{v}$ new master points $\left(x_{v}\right)_{v=1, n, n}$ is given by:

$$
E\left(x_{u}, x_{v}\right)=\int_{\Omega} \frac{\sum_{u=1, n_{u}}\left\|x-x_{u}\right\|^{-2}+\sum_{v=1, n_{v}}\left\|x-x_{v}\right\|^{-2}}{\sum_{v=1}\left\|x-x_{u}\right\|^{-4}+\sum_{v=1, n_{v}}\left\|x-x_{v}\right\|^{-4}} d x
$$

The minimization of $E\left(\left(x_{u}\right)_{u=1, n_{u}},\left(x_{v}\right)_{v=1, n_{v}}\right)$ with respect to $\left(x_{v}\right)_{v=1, n_{v}}$ provides an optimal and consistent way to enrich the existing DOE, with as many master points as desired.

\section{OPTIMIZATION ALGORITHMS}

The meta-model can be utilized either to compute approximations of the objective function within a computationally expensive optimization algorithms, or to build new optimization algorithms based on the sequential improvement of the meta-model itself.

\section{Meta-Model Based Evolutionary Algorithms (MEA)}

The first approach is based on the utilization of Genetic Algorithms (GA) that are quite robust and efficient to find global minima. For each individual of the current population, the value of the objective function is approximated by the meta-model. At each new generation, the meta-model is enriched with $n_{v}$ new points $\left(x_{v}\right)_{v=1, n_{v}}$ in order to better adapt it to the current population $\Omega_{g e n}$. These new points are obtained by minimizing the a priori interpolation error $E_{\Omega_{g e n}}\left(\left(x_{u}\right)_{u=1, n_{u}},\left(x_{v}\right)_{v=1, n_{\nu}}\right)$, where $\left(x_{u}\right)_{u=1, n_{u}}$ is the set of previously utilized master points:

$$
E_{\Omega_{g e n}}\left(\left(x_{u}\right)_{u},\left(x_{v}\right)_{v}\right)=\sum_{i \in \Omega_{g e n}}\left(\tilde{f}_{i}-f_{i}\right)^{2}
$$

In the hybrid algorithm (MEA-H) [14], the quality of the meta-model is enhanced by using the gradient of the objective function, so that $\tilde{f}$ is calculated by equation (5). In the applications, the Pikaiia [15] GA is used with a population of 200 individuals and a limitation to 10 generations. At each new generation, 5 new master points are added to the meta-model, so that 50 calculations are carried out in all.

\section{Sequential Approximation Algorithms} (SAA)

Rather than optimizing the meta-model only for the current population, the meta-model can be sequentially enriched over the full parameter space. The derived Sequential Approximation Algorithm (SAA) follows three steps.

1) The initialization consists in the DOE, i.e. in finding the $n_{v}$ master points that minimize $E\left(\left(x_{v}\right)_{v=1, n_{v}}\right)$, then in evaluating $\left(f_{v}\right)_{v=1, n_{v}}$, and building the corresponding meta-model.

2) The second step is a sequential improvement of the obtained meta-model. Two procedures are used to add one new point to the meta-model at each iteration:

a) selection of the point that minimizes $\tilde{f}-\Delta \tilde{f}$ : it is regarded as the point that has the best potential to minimize $f$.

b) if this point has already been selected in a previous iteration, the position of the new point is given by the DEO, i.e. by minimizing $E\left(\left(x_{u}\right)_{u=1, n_{u}}, x_{v}\right)$ with respect to $x_{v}$ - in practice, it is advisable to select $x_{v}$ points that satisfies $\tilde{f}_{v}-\Delta \tilde{f}_{v} \leq f_{\min }$, where $f_{\min }$ is the calculated minimal value of $f$.

3) The third step is an exploitation of the obtained meta-model. At each iteration, the objective function is evaluated at the point that minimizes $\tilde{f}$, and the meta-model is further enriched as in step 2). However, during this third step, the procedure b) is slightly modified in order to provide a zooming effect: the enrichment of the meta-model is limited to a particular zone of the parameter space, $\Omega_{z o o m}$ - the hyper-sphere centered in $x_{\min }$ (such that $\left.f\left(x_{\min }\right)=f_{\min }\right)$ with such a radius that $(d+1)$ master points are already included into it.

In the applications, the non-gradient interpolation (9) is used for the standard approach (SAA), and the gradient interpolation (5) in the hybrid one (SAA-H). 5 points are generated during the first step, 35 during the second one, and 10 during the last one, so providing a total of 50 functions evaluations. 


\section{Sequential Approximation Algorithms With Uncertainties (SAA-U)}

The use of a meta-model allows taking into account uncertainties on the optimization parameters without significant additional cost, which would not be the case if we were considering uncertainties on other variables such as material or friction data. These uncertainties are assumed to follow a uniform stochastic law of $\Delta x$ range. They are taken into account by replacing $\tilde{f}(x)$ by $\overline{\tilde{f}}(x)$ (16) at any stage of the optimization procedure, so that the sequential improvement of the meta-model goes together with an improvement of the factoring in uncertainties.

$$
\begin{array}{r}
\forall x \in \Omega, \quad \overline{\tilde{f}}(x)=\underset{\bar{x} \in v(x)}{\operatorname{MIN}} \tilde{f}(\bar{x}) \\
\text { with: } \quad v(x)=\left\{\begin{array}{l}
x \in \Omega, \quad \forall i=1, d, \\
x_{i} \in\left[x_{i}-\|\Delta x\|, x_{i}+\|\Delta x\|\right]
\end{array}\right\}
\end{array}
$$

\section{APPLICATIONS}

Table 1 presents the results obtained with different SAAs for different analytical function: camel-back, Rosenbrock, Rastrigin, Grienwanck, and a semi-analytical function - this is a meta-model constructed from a very large number of simulations of the benchmark problem. SAA0 is a variant of SAA where the initial DEO is carried out sequentially, adding values one by one, instead of computing all values simultaneously through the resolution of a higher dimension problem. Table 1 shows that the gradient algorithm SAA0-H allows improving SAA0, but not so significantly. A better initialization with a 5 points DOE, in SAA, provides better results, without the gradient information. For the camel-back function, which is characterized by two minima located in an area with very weak gradients, the introduction of uncertainties allows finding not only a more stable solution, as for the other functions, but also a better one.

For the forging benchmark problem, the new MEA-H and SAA are compared to more standard methods: the BFGS algorithm, the SCPIP algorithm [16], the Meta-model based Evolution Strategy (MES) developed by Emmerich et al. [8] using Kriging interpolation and different algorithmic choices with respect to MEA. As shown in Table 2, the BFGS algorithm is quickly trapped into a local minimum, which prevent it from finding a solution without any folding defect. On the other hand, the SCIP algorithm succeeds to find a much better solution that does not exhibit any fold occurrence. MEA-H finds a more global and better solution. However, the non-gradient MES is able to find an even better one. SAA still provides a better solution, at a significantly different location.

With the introduction of $1 \mathrm{~mm}$ uncertainties on the shape parameters (that are ranging between $-10 \mathrm{~mm}$ and $+20 \mathrm{~mm}$ ), SAA-U allows finding not only a more robust solution but also a globally better one.

\begin{tabular}{|c|c|c|c|c|c|}
\hline & Camel-back & Rosenbrock & Rastrigin & Grienwank & $\begin{array}{c}\text { Semi- } \\
\text { analytical }\end{array}$ \\
\hline SAA0-H & -0.795 & 0.215 & -199.960 & -3.996 & 4.233 \\
\hline $\mathrm{pl}$ & 0.172 & 0.897 & 0.500 & -0.057 & -6.667 \\
\hline $\mathrm{p}^{2}$ & -0.862 & 0.759 & 0.499 & -0.038 & 9.697 \\
\hline SAA0 & -0.847 & 0.643 & -199.980 & -2.773 & 4.622 \\
\hline $\mathrm{p} 1$ & -0.088 & 0.418 & 0.499 & -6.724 & -1.724 \\
\hline $\mathrm{p} 2$ & -0.607 & 0.129 & 0.500 & -0.517 & 14.828 \\
\hline SAA & -0.951 & 0.046 & -199.890 & -4.000 & 4.228 \\
\hline best at iter. & 49 & 47 & 18 & 26 & 49 \\
\hline $\mathrm{pl}$ & 0.120 & 1.009 & 0.498 & 0.002 & -6.711 \\
\hline $\mathrm{p} 2$ & -0.610 & 1.040 & 0.499 & 0.006 & 9.722 \\
\hline SAA-U & -1.0316 & 0.441 & - & - & 4.690 \\
\hline uncertainties & 0.3 & 0.2 & - & - & 0.5 \\
\hline best at iter. & 39 & 46 & - & - & 37 \\
\hline $\mathrm{pl}$ & 0.088 & 0.379 & - & - & -1.506 \\
\hline $\mathrm{p}^{2}$ & -0.710 & 0.120 & - & - & 15.588 \\
\hline Exact/1,000iter. & -1.0316 & 0.000 & -200.000 & -4.000 & - \\
\hline $\mathrm{pl}$ & $-0,0898 / 0,0898$ & 1.000 & 0.500 & 0.000 & - \\
\hline $\mathrm{p} 2$ & $0,7126 /-0,7126$ & 1.000 & 0.500 & 0.000 & - \\
\hline
\end{tabular}

TABLE 1. Results of the various SAA strategies for some analytical and semi-analytical functions. 
TABLE 2. Results of the different tested algorithms for the benchmark spindle optimization problem.

\begin{tabular}{|c|c|c|c|c|c|c|}
\hline Algorithm & Best Value & best at iter. & uncertainties & $\mathrm{p} 1$ & $\mathrm{p} 2$ & Fold \\
\hline BFGS & 10.200 & 7 & - & 6.990 & 18.410 & still present \\
\hline SCPIP & 9.290 & 8 & - & 1.030 & 0.470 & removed \\
\hline MES & 8.140 & 37 & - & -7.870 & -10.000 & removed \\
\hline MEA-H & 9.000 & 20 & - & -5.650 & -7.520 & removed \\
\hline SAA & 8.091 & 51 & - & -9.676 & -9.527 & removed \\
\hline SAA-U & 7.877 & 48 & 1.0 & -8.294 & -8.094 & removed \\
\hline
\end{tabular}

\section{CONCLUSIONS}

Algorithms based on meta-models show quite efficient to solve $3 \mathrm{D}$ complex forging optimization problems within a limited number of iterations. New SAA are quite efficient, and provide better results than the very robust MES that was regarded as a reference. Higher order approximations using the gradient information (SAA0-H) allows improving the algorithm efficiency, but not quite significantly, and a better initialization with a good DOE (SAA approach) shows more effective. Finally, taking into account uncertainties on shape parameters allows escaping some too narrow local minima and finding both a better and more robust solution.

\section{REFERENCES}

1 L. Fourment, J. L. Chenot, Optimal design for nonsteady-state metal forming processes .1. Shape optimization method, Int J Numer Methods Eng $39 \mathrm{n}^{\circ} 1$, (1996) 33-50.

2 S. Badrinarayanan, N. Zabaras, Sensitivity analysis for the optimal design of forming processes, Comp.Meth.Appl.Mech.Eng. 129 February, $n^{\circ} 4$, (1996) 319-348

3 G. Zhao, R. Huff, A. Hutter, R. V. Grandhi, Sensitivity analysis based preform die shape design using the finite element method, J.Materials Engineering and Performance 6 (1997) 303-310.

4 C. F. Castro, L. C. Sousa, C. A. Antonio, J. M. A. César de $\mathrm{Sa}, \mathrm{An}$ efficient algorithm to estimate optimal die shape parameters in forging, Eng. Comput. $18 n^{\circ} 8$, (2001) 1057-1077.

5 S. H. Chung, L. Fourment, J. L. Chenot, S. M. Hwang, Adjoint state method for shape sensitivity analysis in non-steady forming applications, Int J Numer Methods Eng $57 n^{\circ} 10$, , (2003) 1431-1444.
6 M. Laroussi, L. Fourment, The adjoint state method for sensitivity analysis of non-steady problems. application to 3D forging, Int.J.of Forming Processes 7/1-2 (2004) 35-64.

7 L. Fourment, M. Ward, Shape optimization for preform tool design in reverse Superplastic Forming, in: R. Owen, E. Hinton, E. Onate Eds. VII International Conference on Computational Plasticity, 2003.

8 M. Emmerich, A. Giotis, M. Özdemir, T. Bäck, K. Giannakoglou, Metamodel-assisted evolution strategies, Int. Conference on parallel problem solving from nature Springer, Berlin, GERMANY, 2002.

9 D. Buche, N. Schraudolph, P. Koumoutsakos, Accelerating Evolutionary Algorithms with Gaussian Process Fitness Function Models, IEEE Transactions on Systems, Man and Cybernetics 35 (2005) 183-194.

10 M. Bonte, A.H. Van den Boogaard, J. Huetink, A metamodel based optimisation algorithm for metal forming processes. Advanced Methods in Material Forming, D. Banabic (editor), Springer, 55-72 (2007)

11 H. Naceur, S. Ben-Elechi, C. Knopf-Lenoir, J. L. Batoz, Response Surface Methodology for the Design of Sheet Metal Forming Parameters to Control Springback Effects using the Inverse Approach, 2004.

12 L. Fourment, T. T. Do, A. Habbal, M. Bouzaïane, Gradient, non-gradient and hybrid algorithms for optimizing $2 \mathrm{D}$ and $3 \mathrm{D}$ forging sequences, in: $\mathrm{D}$. Banabic Eds.8th International ESAFORM Conference on Material Forming 2005.

13 T. Liszka, J. Orkisz, The finite difference method at arbitrary irregular grids and its application in applied mechanics, Comp.and Struct 11 (1980) 83-95.

14 A. Habbal, L. Fourment, T. T. Tho, Algorithmes hybrides pour l'optimisationglobale. Application en forgeage. Revue Européenne de Mécanique Numérique to be published, (2007)

15 P. Charbonneau, Genetic Algorithms in Astronomy and Astrophysics, The Astrophysical Journal 101 (1995) 309-334.

16 C. Zillober, A combined convex approximation interior point approach for large scale nonlinear programming, Optimization and Engineering 2 (2001) $51-73$. 